\title{
Stress-related coping styles in myalgic university students: A case control study.
}

\section{Xabier Soto-Goñi ( $\nabla$ xabierandersoto@ucm.es )}

Complutense University of Madrid https://orcid.org/0000-0003-4395-3453

\section{Ana Cristina Viñals}

Faculty of dentistry. Complutense University of Madrid

\section{Fabian Pérez-González}

Faculty of dentistry. Complutese University of Madrid

\section{Luis Sánchez-Labrador}

Faculty of dentistry. Complutense University of Madrid

\section{Adelaida Domínguez-Gordillo}

Faculty of dentistry. Complutense University of Madrid

\section{Teresa Sánchez-Sánchez}

Faculty of dentistry. Complutense University of Madrid Ignacio Ardizone-García

Faculty of Dentistry. Complutense University of Madrid

\section{Laura Jiménez-Ortega}

Department of psychobiology. Complutense university of Madrid

\section{Research article}

Keywords: Temporomandibular disorders, psychological factors, coping styles, anxiety, neuroticism, avoidance.

Posted Date: April 15th, 2020

DOl: https://doi.org/10.21203/rs.2.15856/v4

License: (c) (1) This work is licensed under a Creative Commons Attribution 4.0 International License. Read Full License 


\section{Abstract}

Background: Patients suffering pain-related temporomandibular disorders (TMD) exhibit greater levels of psychological distress, environmental stress, somatic symptoms, anxiety, depression, somatic awareness, pain catastrophizing, and impaired pain coping strategies compared to pain-free controls. However, little is known about psychological factors involved in the different TMD types fulfilling DC/TMD criteria. Furthermore, regardless of severity, the role of general coping strategies and styles in TMD is not yet well understood. The main goal of this study was to investigate stress-related coping styles, anxiety and personality traits in a group of dentistry students suffering from temporomandibular disorder with myalgia.

Methods: A cohort of 102 university students was initially recruited for this study. Following clinical evaluation, a myalgia group (24 participants) and a control group (25 participants) were formed. Participants were later assessed in anxiety, stress coping strategies, and personality measures by using the State-trait anxiety inventory (STAI), coping response inventory (CRI), and Neo Five-Factor Inventory (NEO-FFI) questionnaires respectively.

Results: The myalgia group presented greater levels of trait anxiety and neuroticism in comparison to the control group. Participants with myalgia also showed higher levels of avoidance coping which was the only reliable predictor of TMD.

Conclusions: Avoidance coping strategies are generally considered maladaptive, as they seem to increase perceived stress, a robust predictor of TMD. Interventions aimed at reducing stress and anxiety levels, increase emotional stability, and preventing maladaptive coping styles, might improve temporomandibular health and prevent the myalgia and its chronification.

\section{Background}

According to the American Academy of Orofacial Pain's (AAOP) definition, Temporomandibular Disorders (TMD) include a group of musculoskeletal and neuromuscular pathological conditions that involve the Temporomandibular joint (TMJ), the masticatory muscles and all associated tissues [1]. While patients with these disorders are also characterized by limited jaw movement and TMJ joint sounds, like clicks or crepitation, the most common complaint is pain, usually localized in the masticatory muscles and the preauricular area. TMD is thought to affect from 10 percent to 30 percent of individuals globally. However, only in $15 \%$ of cases, individuals perceive that they need treatment [2]. TMD refers to a group of heterogeneous conditions that can be divided into 2 main subgroups: Temporomandibular joint disorders and masticatory muscle disorders. Among the disorders of the masticatory muscles, myalgia (which includes local myalgia, myofascial pain, and myofascial pain with referral) is one of the most frequent in the general population [3].

Despite the fact that some studies find somatic symptoms to be more strongly associated with TMD onset, psychological variables such as perceived stress, previous life evets and negative effect have also 
been shown to be significantly associated and predictive of TMD [4]. Thus, currently, TMD cannot be understood without taking into account the biopsychosocial framework [5]. Although few studies have explored psychological factors in TMD subtypes, a positive relationship between anxiety, depression and somatization with chronic myofascial pain (a subtype of myalgia) has been observed [6]. Furthermore, several studies suggest that certain psychological factors can predispose an individual to TMD $[7,8]$. Thus, the well-known OPPERA cohort study revealed that somatic symptoms, perceived stress, negative mood and personality traits such as the neuroticism predicted an increased risk of TMD first-onset [4]. In this line, increased risk of developing TMD is more pronounced for individuals whose genetic susceptibility increases responsiveness to catecholamine neurotransmitters [9] which are involved in the stress response. However, it has also been shown that painful TMDs can also relate to altered clinical, psychological and biological factors. Thus, according to the literature, TMD patients experiencing pain present greater levels of psychological distress, environmental stress, somatic symptoms, anxiety, depression, somatic awareness, and pain catastrophizing compared to pain-free controls [10-15]. Therefore, a two-way relationship between TDM and psychological factors has been proposed [16].

The relationship between pain coping, specifically catastrophizing, and TMD severity has been well investigated [17]. However, the relationship between stress-related coping and TDM is not yet well understood and particularly myalgia TMD $[18,19,20]$. Generally, stress-related coping can be defined as the predictable cognitive and behavioral efforts to manage environmental and internal demands or conflicts. Although, data point out that stress-related coping influences perceived stress [21], the different approaches and models about stress-related coping styles make it difficult to compare findings and extract clear conclusions. Some authors differentiate problem-focused and emotion-focused coping strategies [21], while others distinguish between approach-versus avoidance-oriented coping [22], adaptive versus maladaptive coping [23], and active versus passive coping [24,25]. However, in general, it seems problem-focused, active coping and approach oriented coping can be regarded as adaptive, whereas emotion-focused, passive and avoidance-oriented coping can be considered maladaptive [18].

Taking the later in account, previous research pointed out that people suffering TMD showed more maladaptive stress-related coping responses and less adaptive ones in comparison to controls $[18,19,20]$. Specifically, TMD patients use more escape-avoidance strategies [20], less problem solving strategies [19], minor use of positive reinterpretation and humor as coping strategies, and show lower interest in seeking instrumental social support [19]. Studies have confirmed that a passive attitude, exaggeration of negative consequences, and reduced use of distraction strategies constitute a dysfunctional style of coping, which is associated with greater levels of distress in TMD patients [17]. Therefore, maladaptive coping might lead to an increase of perceived stress and consequently to an increased risk of suffering TMD.

Furthermore, oral health-related quality of life in myalgic patients appeared to worsen with coping strategies that reveal passive or avoidant attitudes of the patient [26]. In contrast, it has also been reported that TMD patients with pain primarily in the masticatory muscles score higher on the active coping scale than patients with arthrogenic pain, $[27,28]$. Altogether, this data shows that it is not jet well 
understood the relation between stress coping and TMD, particularly in myalgic patients, calling for further study.

Indeed, different perspectives and assessments regarding coping have led to a variety of results and interpretations. The relationship between pain coping and TMD has been well explored [17]. However, stress-related coping has been less investigated. Nonetheless, perceived stress, which is a strong predictor of TMD [4], is negatively related to adaptive coping strategies. These results are in line with the classical model of Lazarus and Folkman [21], in the sense that the way people perceive a stress situation might be related with their coping capabilities. Taking all together, it seems necessary to clarify the relationship between TMD and stress-related coping styles. In addition, most studies investigating coping behavior are focused on just one dimension of coping (such as active versus passive coping, or approach versus avoidance behavior), while few studies explore multiple coping responses and styles.

Furthermore, only a few studies have considered the role of psychological factors when taking into account the different TMD types according to the DC/TMD criteria. Few of them have focus either on arthralgia or myalgia [6], while previous evidences indicate that the role of coping styles and psychological factors might be different for these TMD types $[19,28]$. A more systematized approach, using valid and reliable coping questionnaires able to explore and quantify several coping strategies, is needed to clarify the role of stress-related coping in TMD, particularly in myalgia, the TMD type in which psychological factors and coping styles might be more involved, when compared to other TMD types $[26,29]$.

This study aims to study stress-related coping style in depth (see Table 1 for scales), the levels of anxiety and personality factors in a group of university students presenting myalgia, according to the DC/TMD, in comparison to a control group free of symptoms. It is hypothesized that the way in which people face and perceive their problems is relevant in the development of myalgia, therefore it is expected that the myalgia group and the control group will differ significantly in terms of their coping strategy pattern, anxiety and neuroticism.

Table 1: Coping Response Inventory (CRI) scales [33] according to coping dimensions. Examples are given base on CRI specific questions.

APPROACH COPING

Logical Analysis

e.g. Thinking about different solutions

COGNITIVE COPING

BEHAVIORAL COPING
Positive Reappraisal

e.g. Trying to see the bright side of the problem

Seeking Guidance and Support

e.g. getting help of expert counselors

Problem Solving

e.g. Deciding on and implementing a solution
AVOIDANCE COPING

Cognitive Avoidance

e.g. Avoiding thinking about the problem

Acceptance or Resignation

e.g. Accepting that nothing can be done.

Seeking Alternative Reward e.g. Looking for alternative distractions.

Emotional Discharge e.g. Crying, grieving 


\section{Methods}

A total of 102 volunteer students from the Faculty of Odontology at Complutense University of Madrid, both male ( $\mathrm{N}=25)$ and female $(\mathrm{N}=77)$ ranging from 18-35 years old (mean age 21), were recruited for this study. Participants with active orthodontic or psychopharmacological treatment were excluded from this study. Participants were included in the study after giving their written informed consent according to the Declaration of Helsinki and approved by the ethics committee of the "Hospital Clínico Universitario", UCM, Madrid, Spain (Reference: 12/043-E)". Data from a total of 49 participants was analyzed, as the other 53 participants did not meet the classification criteria for the myalgia group but presented some minor sign/symptoms or were excluded in order to match demographic variables (gender, age, ethnicity, etc) between groups. That is to say, control group was composed of healthy participants that did not present any signs or symptoms of any TMD type and case group was composed by participants that clearly fulfilled DC/TMD criteria for myalgia.

In order to evaluate the participants' oral history, they were administered the TMD Pain Screener and the Symptom Questionnaire by the Diagnostic Criteria (DC/TMD) Axis I evaluation [3]. A questionnaire regarding demographic data was also administered (i.e. gender, age, ethnicity, family income).

After completing the questionnaires related to participants' oral history, two dentists specialized in TMD performed oral examinations of TMD signs and symptoms using the DC/TMD Examination protocol. Dentists were adequately trained in the DC/TMD protocol at the "temporomandibular disorders and orofacial pain" magister (University Complutense of Madrid). A preliminary calibration was performed between the 2 dentists to check the inter-rater reliability, resulting in a good degree of agreement, expressed by a Kappa index of 0.76 . The examination included TMJ palpation, masticatory muscles palpation and assessment of opening movement patterns and joint sounds like clicks or crepitation.

Once signs and symptoms were assessed, two groups of participants were formed, matched in terms of demographic variables. The myalgia group ( $\mathrm{N}=24,4$ males) included students that were clinically diagnosed with myalgia, and was further subdivided into three DC/TMD types: (1) local myalgia ( $N=5)$, defined by the DC/TMD as "pain localized to the site of palpation"; (2) myofascial pain ( $N=16)$, defined as "pain spreading beyond the site of palpation but within the boundary of the muscle being palpated"; and (3) myofascial pain with referral $(\mathrm{N}=3)$, defined as "pain at a site beyond the boundary of the muscle being palpated"[3]. Only 3 out of 24 myalgic participants have request professional help and used occlusal splint regularly. The control group ( $\mathrm{N}=25,7$ males) consisted of students who did not show any signs nor symptoms of any type of TMD.

Following the oral examinations, participants were called back two weeks later to complete the psychological questionnaires. Stressful periods like examination dates were avoided during data collection.

The evaluation of psychological factors included anxiety, stress coping and personality measurements. Anxiety was assessed using the State-trait anxiety inventory (STAI), which includes 20 items for 
assessing trait anxiety (STAI-T) and 20 for assessing state anxiety (STAI-S) [30]. Stress coping was measured using the Coping Responses Inventory-Adult Form (CRI), which contains 48 items grouped into 8 scales: Logical Analysis, Positive Reappraisal, Seeking Guidance and Support, Problem Solving, Cognitive Avoidance, Acceptance or Resignation, Seeking Alternative Rewards and Emotional Discharge. These 8 scales are classified according to two dimensions identified in the literature [31]: Cognitive and Behavioral coping on the one hand, and Approach vs. Avoidance coping on the other hand (see Table 1). Lastly, personality was assessed by the Neo Five-Factor Inventory (NEO-FFI), a 60-item measure of the five major domains of personality: Neuroticism, Extraversion, Openness to Experience, Agreeableness, and Conscientiousness. Evidence of convergent and discriminant validity is presented in the NEO-PI-R manual. [32]. All selected tests are validated for a Spanish sample (Spain), are commonly used in research [33] and have high levels of reliability (Cronbach alphas between 0,59 and 0,93 ) and validity (correlations generally above 0,70 ). A blind correction of the questionnaires was carried out by two psychologists specialized in health psychology and psychological assessment, who were blind to the experimental conditions.

Statistical analyses were calculated using SPSS 21 Statistics Software (IBM) and R, including the package MVN for Mardia's multivariate analysis [34]. First at all, data were standardized by calculating the t-scores $(c=50, s d=10)$ of each scale within each questionnaire. Items multivariate normality was assessed via Mardia's multivariate kurtosis and skewness coefficients [35]. Second, one-way multiple variable analyses of variance (MANOVA) was performed, where the group (myalgia vs controls) was the intersubject factor and the dependent variables were the scales of each test. To fulfill the assumption of independence, a second MANOVA was calculated, excluding CRI strategies, but including the type of strategy (Cognitive, Behavioral, Approach, and Avoidance coping), since the later are combinations of different strategies (see Table 1). This type of analyses was chosen, because one of its benefits is to control for Type 1 error than might occur by conducting multiple t-tests. Furthermore, effect size (partial eta squared: $\otimes^{2} p$ ) and power $(\pi)$ were calculated for each variable included in the MANOVA.

\section{Results}

\section{MANOVA Analyses}

The estimates of Mardia's multivariate kurtosis and skewness coefficients were non-significant, $0.01, p=$ .994 , and $721.37, \mathrm{p}=.132$ respectively, therefore granting a parametric MANOVA for the analyses.

In regard to anxiety measurements, results from the one-way MANOVA revealed higher levels of trait anxiety in the myalgia group in comparison to the control group $\left(F_{(1,47)}=4.46 p=.04, \rrbracket^{2} p=.087, \pi=.544\right)$ (see Figure 2), while for state anxiety no significant effects were observed (see Table 2 for detailed results including non-significant ones)

Additionally, analyses of NEO-FFI data indicated that the level of neuroticism was, on average, higher for the myalgia group in comparison to the control group $\left(\mathrm{F}_{(1,47)}=6.39 p=.015, \mathbb{\nabla}^{2} p=.120, \pi=.698\right)$. No 
significances were observed in the other variables of this questionnaire (see Figure 3)

Lastly, with respect to coping measurements, the findings showed that the myalgia group presented significantly higher levels of Acceptance and Resignation $\left(F_{(1,47)}=4.21 p=.046, \nabla^{2} p=.082, \pi=.521\right)$ and Seeking Alternative Reward $\left(F_{(1,47)}=5.69 p=.021, \otimes^{2} p=.108, \pi=.647\right)$ in comparison to the control group. Regarding the type of strategy, to fulfill independence assumption, a separate MANOVA including type of strategies (Cognitive, Behavioral, Approach, and Avoidance coping), but not the strategies themselves was calculated. Significant differences were observed exclusively for Avoidance coping $\left(\mathrm{F}_{(1,47)}=8.58 p\right.$ $\left.=.005, \nabla^{2} p=.154, \pi=.819\right)$. Overall, the myalgia group presented larger use of avoidance strategies than the control group. No significances were observed in the other variables of this questionnaire (see Figure 4)

Table 2: STAI, NEO and CRI multiple analyses of variances (MANOVAs) including effect size and power. Average percentiles for university students have been also included according to the questionnaire's manual [32,33,34]. 


\begin{tabular}{|c|c|c|c|c|c|c|c|}
\hline Group & Mean & $\begin{array}{l}\text { Standard } \\
\text { desviation }\end{array}$ & $\begin{array}{c}\text { Percentile } \\
\text { score }\end{array}$ & $F_{(1,47)}$ & Sig. & $\begin{array}{c}\text { Effect } \\
\text { size }(\mathbb{p} \text { p) }\end{array}$ & Power (II) \\
\hline
\end{tabular}

\begin{tabular}{|c|c|c|c|c|c|c|c|c|}
\hline \multirow[t]{2}{*}{ Anxiety Trait } & Myalgia & 48.82 & 8.91 & 55 & \multirow[t]{2}{*}{4.46} & \multirow[t]{2}{*}{.04} & \multirow[t]{2}{*}{.087} & \multirow[t]{2}{*}{.544} \\
\hline & Control & 44.38 & 5,44 & 30 & & & & \\
\hline \multirow[t]{2}{*}{ Anxiety state } & Myalgia & 47.32 & 7.31 & 50 & \multirow[t]{2}{*}{1.46} & .23 & .030 & .220 \\
\hline & Control & 44.94 & 6.39 & 40 & & & & \\
\hline \multirow[t]{2}{*}{ NEO - Neuroticism } & Myalgia & 53.12 & 2.29 & 60 & \multirow[t]{2}{*}{6.39} & .01 & .120 & .698 \\
\hline & Control & 51.64 & 1.8 & 55 & & & & \\
\hline \multirow[t]{2}{*}{ NEO - Extraversion } & Myalgia & 50.58 & 3.71 & 50 & \multirow[t]{2}{*}{.37} & .54 & .008 & .092 \\
\hline & Control & 51.12 & 2.27 & 55 & & & & \\
\hline \multirow[t]{2}{*}{ NEO - Openness } & Myalgia & 50.21 & 2.34 & 50 & \multirow[t]{2}{*}{1.05} & .31 & .022 & .171 \\
\hline & Control & 49.48 & 2.61 & 45 & & & & \\
\hline \multirow[t]{2}{*}{ NEO - Agreeableness } & Myalgia & 47.08 & 4.43 & 40 & \multirow[t]{2}{*}{2.7} & .11 & .054 & .364 \\
\hline & Control & 48.76 & 2.47 & 45 & & & & \\
\hline \multirow[t]{2}{*}{ NEO - Conscientiousness } & Myalgia & 48.75 & 2.64 & 45 & \multirow[t]{2}{*}{.005} & .94 & .000 & .051 \\
\hline & Control & 48.8 & 2.38 & 45 & & & & \\
\hline \multirow[t]{2}{*}{ CRI - Logical Analysis } & Myalgia & 49.45 & 9.64 & 45 & \multirow[t]{2}{*}{1.65} & .20 & .034 & .243 \\
\hline & Control & 46.36 & 7.07 & 35 & & & & \\
\hline \multirow[t]{2}{*}{ CRI - Positive Reappraisal } & Myalgia & 50.08 & 9.01 & 50 & \multirow[t]{2}{*}{.000} & .98 & .000 & .050 \\
\hline & Control & 50.04 & 7.45 & 50 & & & & \\
\hline \multirow{2}{*}{$\begin{array}{l}\text { CRI - Seeking Guidance } \\
\text { and Support }\end{array}$} & Myalgia & 52.46 & 11.21 & 60 & \multirow[t]{2}{*}{.029} & .86 & .001 & .053 \\
\hline & Control & 53 & 10.9 & 60 & & & & \\
\hline \multirow[t]{2}{*}{ CRI - Problem Solving } & Myalgia & 49.92 & 7.92 & 50 & \multirow[t]{2}{*}{1.01} & .32 & .021 & .167 \\
\hline & Control & 47.28 & 10.19 & 40 & & & & \\
\hline \multirow[t]{4}{*}{ CRI - Cognitive Avoidance } & Myalgia & 53.21 & 9.01 & 60 & \multirow[t]{2}{*}{1.65} & .20 & .034 & .242 \\
\hline & Control & 49.68 & 10.14 & 50 & & & & \\
\hline & Myalgia & 56.67 & 12.39 & 70 & \multirow[t]{2}{*}{4.21} & .04 & .082 & .521 \\
\hline & & & Page $\varepsilon$ & & & & & \\
\hline
\end{tabular}


CRI- Acceptance or

Resignation

50.12

\begin{tabular}{|c|c|c|c|c|c|c|c|c|}
\hline \multirow{2}{*}{$\begin{array}{l}\text { CRI - Seeking Alternative } \\
\text { Reward }\end{array}$} & Myalgia & 52.92 & 14.89 & 60 & \multirow[t]{2}{*}{5.69} & \multirow[t]{2}{*}{.02} & \multirow[t]{2}{*}{.108} & \multirow[t]{2}{*}{.647} \\
\hline & Control & 44.64 & 8.71 & 30 & & & & \\
\hline \multirow[t]{2}{*}{ CRI - Emotional Discharge } & Myalgia & 50.75 & 8.54 & 50 & \multirow[t]{2}{*}{1.54} & .22 & .032 & .230 \\
\hline & Control & 47.92 & 7.36 & 40 & & & & \\
\hline \multirow[t]{2}{*}{ Cognitive coping } & Myalgia & 52.35 & 6.72 & 60 & \multirow[t]{2}{*}{3.76} & .058 & .074 & .477 \\
\hline & Control & 49.05 & 5.11 & 45 & & & & \\
\hline \multirow[t]{2}{*}{ Behavioral coping } & Myalgia & 51.51 & 7.13 & 55 & \multirow[t]{2}{*}{3.97} & .052 & .078 & .497 \\
\hline & Control & 48.21 & 4.11 & 45 & & & & \\
\hline \multirow[t]{2}{*}{ Approach coping } & Myalgia & 50.48 & 6.86 & 50 & \multirow[t]{2}{*}{.55} & .46 & .012 & .113 \\
\hline & Control & 49.17 & 5.41 & 45 & & & & \\
\hline \multirow[t]{2}{*}{ Avoidance coping } & Myalgia & 53.38 & 6.98 & 60 & \multirow[t]{2}{*}{8.6} & .005 & .154 & .819 \\
\hline & Control & 48.09 & 5.61 & 45 & & & & \\
\hline
\end{tabular}

\section{Logistic regression}

Logistic regression analyses were then conducted to examine the interdependence of Anxiety-trait, Neuroticism, and Avoidance coping variables in their association with TMD. The full model was significantly reliable $\left(X^{2}(3)=11,73, P=, 008\right)$, this model accounted for between 21.3 and 28.4 of the variance in TMD status with the $74,5 \%$ of the participants successfully predicted. Only Avoidance coping significantly predicted TMD status (odds ratio $=1.12,95 \% \mathrm{Cl}=1.08-1.16, \mathrm{P}=.03$ ).

\section{Discussion}

Out of 102 university students initially considered, 24 of them suffered from myalgia (myalgia group) and 25 participants did not have any TMD-related symptom of signs (control group). When comparing anxiety, personality and coping variables between groups, the myalgia group presented greater levels of trait anxiety and neuroticism in comparison to the control group. In addition, participants with myalgia also showed higher levels of Acceptance and Resignation as well as Seeking Alternative Rewards, and in general they used significantly more often avoidance coping strategies, which was the only reliable predictor of DTM according to the logistic regression model. 
The simplest result of this study is that out of the 102 individuals initially considered, 24 of them suffered from myalgia which formed the myalgia group, although the majority of these participants did not consider it severe enough to request professional help. Only 25 participants did not present any TMDrelated symptom or signs. It should be noted that the participants were university students (a specific subgroup of young adults) and a more representative sample would have increased the study validity. Nevertheless, the percentage of students suffering TMD matches population prevalence suggested by other studies $[15,36,37,38,39]$. Furthermore, participants average score of the psychological questionnaires were similar to other Spanish university students (Spain) according to norms provided by questionnaire manuals [30,31,32], since percentiles where within 40 and 60 (see Table 2), except for anxiety trait and just one of the CRI coping strategy, logical analyses (30 and 35 , respectively). In addition, the sample selection (a cohort of university students) favored the homogeneity of the samples in terms of age, sociological, cultural and environmental variables. Nonetheless, it should be taken into account that even though the myalgia group clearly fulfilled the diagnostic criteria, they weren't severe patients seeking consultation. The fact that psychological alterations are already present in non-severe patients would support their importance in chronic and more severe TMD patients.

In accordance with previous studies in TMD, the myalgia group showed higher levels of trait anxiety than the control group $[4,6,10-12,40]$ However, while the OPPERA prospective cohort study detected significantly larger levels of trait and state anxiety for TMD patients [4], in the present study state anxiety differences were not significant. This might be due to a high level of homogeneity between the myalgia and control groups, a cohort of university students, which were probably facing similar social and environmental demands. Alternatively, this could also be due to a lack of power resulting from the number of participants, since data analyses for anxiety scores revealed small size effects and powers. In addition, the present sample included participants suffering exclusively from myalgia. According to the present data, higher anxiety levels in myalgia might appear mainly because of the individuals' traits, regardless of the situation, at least for young adult students.

A relationship between TMD and the Neuroticism personality trait has been previously observed [41]. In the same line the OPPERA study showed that it is at least a predictor of TMD [4], however it did not differentiate between articular and muscular TMD pathologies. In the studies in which the differences between muscular TMD and articular TMD are analyzed, differences appear. Thus, Ferrando et al. [19] showed that myofascial patients present higher levels of neuroticism, whereas conscientiousness and self-discipline were higher in the articular group. Similarly, the present findings show that myalgic participants have larger levels of neuroticism than controls. It should be considered that personality is a psychological construct developed during the childhood-adolescence which is quite stable throughout life [42], therefore it might be scarcely affected by the curse of a disease. Taken the later in account, these results suggest that neuroticism might be an important personality factor contributing to TMD, at least for myalgia. Nonetheless, although neuroticism is often considered a stable personality trait depending on genetic and environmental factors [43], emotionally focused therapies, such as the unified protocol for the treatment of emotional disorders [44], might be useful to prevent TMD. 
Coping styles were thoroughly investigated by means of the CRI inventory in the present study. The myalgia group used avoidance coping strategies significantly more often. Particularly, Acceptance and Resignation as well as Seeking Alternative Rewards were more prevalent in myalgia group than in the control group. Furthermore, according to the logistic regression model, Avoidance Coping is the best and only significant predictor of TMD. Therefore, it could be said that myalgic patients, in comparison to controls, tend to face a problem by looking for distractions, avoiding the problem and accepting that they can do nothing to solve it. Avoidance coping strategies are often regarded as maladaptive. Furthermore, maladaptive coping is related to depression and anxiety, [45]. TMD patients seem to cope with stress differently than individuals from the general population. In line with the present findings, an increase of escape-avoidance strategies for TMD patients has been previously described, which were interpreted as maladaptive [18,20]. In this line, a recent study found that avoidance and passive coping strategies might worsen oral health-related quality of life in patients with myalgia [26]. In contrast, some studies have observed higher levels of active coping in masticatory muscle pain patients in comparison with articular pain patients $[27,28,46]$, although a symptomless control group was not included, making comparisons harder to establish. In contrast to previous research, the present study did not identify reduced adaptive coping strategies in the myalgia group. It should also be considered, however, that the participants were young students presenting myalgia, unlike previous studies, where the lack of adaptive coping strategies might well appear as a consequence of long lasting and more severe TMD and/or aging. Nonetheless, the differences in the assessment and definition of coping strategies across the few studies in the literature that assess coping in TMD make similarities hard to establish.

The way people face problems or stressful situations and the way in which they interpret them, may play a role in TMD, particularly in myalgia. Our data appears compatible with this hypothesis, as it indicated larger levels of avoidance/maladaptive coping styles in myalgic patients in comparison to the control group. In addition, it was the only reliable predictor of TMD according to the logistic regression model. The well-known OPPERA study investigated pain coping, but not stress-related coping strategies. however, they established that perceived stress increased the risk of first-onset TMD [4,46,47]. Specifically, maladaptive coping strategies might increase perceived stress, a strong predictor of TMD $[48,49]$, thus playing a role in TMD. Therefore, intervention programs including techniques to promote the use of adaptive coping strategies, reduce maladaptive ones and foster emotional stability, which is related with neuroticism trait, may be useful to prevent the development of temporomandibular myalgia.

The present study points out that coping styles might be related with myalgia, although several limitations should be addressed in future research. A sample of dentistry students guarantees, on the one hand, the accuracy of symptoms detection (because of their symptoms awareness, and their knowledge about them), and on the other hand, it also equalized, educational level, similar environment and lifestyle between myalgic participants and controls. However, as discussed above, case group included young students presenting myalgia, even though they clearly fulfilled the diagnostic criteria, they weren't severe patients seeking consultation. Therefore, further research including patients and a larger and more heterogenic sample of people could enhance the generalizability of the results. In addition, psychological 
factors, including depression, emotional intelligence and catastrophizing among others, should be investigated taking into account the TMD subtypes proposed by the new DC/TMD classification.

\section{Conclusions}

University students diagnosed with myalgia showed higher levels of neuroticism, trait anxiety, and avoidance coping style in comparison to the symptomless individuals in the control group. In light of the results and considering that avoidance coping was the only reliable predictor of TMD and it is related to the increase of perceived stress, interventions to reduce anxiety and stress levels, together with maladaptive coping styles management and prevention, might improve temporomandibular health. In addition, taking in account the larger neuroticism levels, emotionally focused therapies, such as the unified protocol for the treatment of emotional disorders, might also help prevent TMD. However, further research is required in myalgia which includes other psychological factors, such as emotional intelligence, in larger and more heterogeneous samples.

\section{List Of Abbreviations}

TMD: Temporomandibular disorders

TMJ: Temporomandibular joint

DC/TMD: Diagnostic criteria of temporomandibular disorders

AAOP: American Academy of Orofacial Pain

OPPERA study: Orofacial pain prospective evaluation and risk assessment study

STAl: State-Trait Anxiety Inventory

NEO-FFI: Neo Five-Factor Inventory

CRI: Coping Responses Inventory

MANOVA: Multivariate analysis of variance

\section{Declarations}

\section{Ethics approval and consent to participate}

Participants were clinically evaluated after giving their written informed consent according to the Declaration of Helsinki, and approved by the ethics committee of the Hospital Clínico Universitario, UCM, Madrid, Spain (Reference: 12/043-E).

Consent for publication 
Not applicable

\section{Availability of data and materials}

The datasets used and/or analyzed during the current study are available from the corresponding author on reasonable request

\section{Competing interests}

All authors declare there are non-financial competing interests (political, personal, religious, ideological, academic, intellectual, commercial or any other) in relation to this manuscript.

\section{Funding}

Not applicable

\section{Authors' contributions}

XSG, ACV and LJO conceived and designed the study, performed the questionnaires correction, statistical analysis, participated in the interpretation of the data, and the manuscript drafting.

FPG and LSL participated in the data collection

ADG, TSS and IAG, conceived and designed the study and collaborated in the interpretation of the data

\section{Acknowledgements}

The authors are very thankful to Complutense University of Madrid, particularly to magister of specialist in temporomandibular disorder and orofacial pain.

\section{References}

1. Fernández-de-las-Penas C, Svensson P. Myofascial Temporomandibular Disorder. Curr Rheumatol Rev. 2016;12(1):40-54.

2. Kelleher M, Ray-Chaudhuri A, Khawaja N. Patients' Priorities and Attitudes Towards Their TemporoMandibular Disorders. Primary Dental Journal. 2015;4(3): 17-21.

3. Schiffman E, Ohrbach R, Truelove E, Look J, Anderson G, Goulet JP, ... Dworkin SF. Diagnostic Criteria for Temporomandibular Disorders (DC/TMD) for Clinical and Research Applications: recommendations of the International RDC/TMD Consortium Network and Orofacial Pain Special Interest Group. Journal of Oral \& Facial Pain and Headache. 2014;28(1):6-27.

4. Fillingim RB, Ohrbach R, Greenspan JD, et al. Psychological factors associated with development of TMD: the OPPERA prospective cohort study. J Pain. 2013 Dec;14 Suppl 12:75-90. 
5. Darnall B, Carr D, Schatman Pain Psychology and the Biopsychosocial Model of Pain Treatment: Ethical Imperatives and Social Responsibility. Pain Medicine. 2016;18.

6. Velly AM, Gornitsky M, Philippe P. Contributing factors to chronic myofascial pain: a case-control study. J Pain. 2003;104(3), 491-499.

7. Bonjardim LR, Gavião MBD, Pereira LJ, et al. Anxiety and depression in adolescents and their relationship with signs and symptoms of temporomandibular disorders. Int J Prosthodont. 2005;18(4):347-352.

8. Celić R, Pandurić J, Dulcić N. Psychologic status in patients with temporomandibular disorders. Int J Prosthodont. 2006;19(1):28-29.

9. Slade GD, Sanders AE, Ohrbach R, et al. COMT Diplotype Amplifies Effect of Stress on Risk of Temporomandibular Pain. J Dent Res. 2015 Sep; 21;94(9):1187-1195.

10. Carlson CR, Okeson JP, Falace DA, et al. Comparison of psychologic and physiologic functioning between patients with masticatory muscle pain and matched controls. J Orofac Pain. 1993;7(1):1522.

11. Fillingim RB, Ohrbach R, Greenspan JD, et al. Potential psychosocial risk factors for chronic TMD: Descriptive data and empirically identified domains from the OPPERA case-control study. J Pain. 2011 Nov;12 Suppl 11:T46-60.

12. Quartana PJ, Buenaver LF, Edwards RR, et al. Pain catastrophizing and salivary cortisol responses to laboratory pain testing in temporomandibular disorder and healthy participants. J Pain. 2010 Feb;11(2):186-194.

13. Gatchel RJ, Peng YB, Peters ML, et al. The biopsychosocial approach to chronic pain: scientific advances and future directions. Psychol Bull. 2007 Jul; 133(4):581-624.

14. Keefe FJ, Rumble ME, Scipio CD, et al. Psychological aspects of persistent pain: current state of the science. J Pain. 2004 May;5(4):195-211.

15. Fernandes G, van Selms MKA, Gonçalves DAG, et al. Factors associated with temporomandibular disorders pain in adolescents. J Oral Rehabil. 2015 Feb; 42(2):113-119.

16. Fillingim RB, Slade GD, Greenspan JD, et al. Long-term changes in biopsychosocial characteristics related to temporomandibular disorder: findings from the OPPERA study. Pain. 2018;159(11): 24032413.

17. Turner JA, Dworkin SF, Mancl L, et al. The roles of beliefs, catastrophizing, and coping in the functioning of patients with temporomandibular disorders. Pain. 2001 May; 92(1-2):41-51. 
18. Reissmann DR, John MT, Schierz O, et al. Stress-related adaptive versus maladaptive coping and temporomandibular disorder pain. J Orofac Pain. 2012;26(3):181-190.

19. Ferrando M, Andreu Y, Galdón MJ, et al. Psychological variables and temporomandibular disorders: distress, coping, and personality. Oral Surg Oral Med Oral Pathol Oral Radiol Endod. 2004 Aug;98(2):153-160.

20. Callahan CD. Stress, coping, and personality hardiness in patients with temporomandibular disorders. Rehabil Psychol. 2000;45(1):38-48.

21. Lazarus RS FS. Stress, Appraisal and Coping. Psychol Med. 1985 Aug; 9;15(03):705.

22. Suls J, Fletcher B. The relative efficacy of avoidant and nonavoidant coping strategies: a metaanalysis. Health Psychol. 1985;4(3):249-288.

23. Tan G, Teo I, Anderson KO, et al. Adaptive Versus Maladaptive Coping and Beliefs and Their Relation to Chronic Pain Adjustment. Clin J Pain. 2011;27(9):769-774.

24. Gatchel R, Turk D. Psychological Approaches to Pain Management: A Practitioner's Handbook. J Psychosom Res. 2006; 42.

25. Brown GK, Nicassio PM. Development of a questionnaire for the assessment of active and passive coping strategies in chronic pain patients. Pain. 1987 Oct; 31(1):53-64.

26. Hasanoglu Erbasar GN, Alpaslan C. Influence of coping strategies on oral health-related quality of life in patients with myalgia. Cranio. 2009; 37(2): 94-100.

27. Kight M, Gatchel RJ, Wesley L. Temporomandibular disorders: evidence for significant overlap with psychopathology. Health Psychol. 1999 Mar;18(2):177-182.

28. Galdon MJ, Durá E, Andreu Y, et al. Multidimensional approach to the differences between muscular and articular temporomandibular patients: coping, distress, and pain characteristics. Oral Surg Oral Med Oral Pathol Oral Radiol Endod. 2006 Jul;102(1):40-46

29. McCreary CP, Clark GT, Merril RL, Flack V, Oakley ME. Psychological distress and diagnostic subgroups of temporomandibular disorder patients. Pain. 1991;44(1):29-34.

30. Spielberger CD, Gorsuch RL, Lushene RE, et al. STAI: Cuestionario de ansiedad estado-rasgo. 9th ed. Madrid: Tea ediciones; 2011.

31. Moos RH. CRI-A: Inventario de respuestas de afrontamiento para adultos. Madrid: Tea ediciones; 2010

32. Costa PT, McCrae RR. Inventario de Personalidad Neo - Revisado (NEO-PI-R) e Inventario Neo Reducido de cinco factores (NEO-FFI). Madrid: Tea Ediciones; 2008. 
33. Bartkowska W, Samborski W, Mojs E. Cognitive functions, emotions and personality in woman with fibromyalgia. Anthropol Anz. 2018 Dec; 11;75(4):271-277

34. Korkmaz S, Goksuluk D, Zararsiz G. MVN: An R package for assessing multivariate normality. The R Journal. 2014; 6(2):151-162.

35. Mardia KV. Measures of multivariate skewness and kurtosis with applications. 1970;57(3):519-530.

36. Calixtre LB, Grüninger BL da S, Chaves TC, et al. Is there an association between anxiety/depression and temporomandibular disorders in college students? J Appl Oral Sci. 2014 Jan-Feb; 22(1):15-21.

37. Lövgren A, Österlund C, Ilgunas A, Lampa E, Hellström F. A high prevalence of TMD is related to somatic awareness and pain intensity among healthy dental students. Acta Odontologica Scandinavica. 2018;76(6):1-7.

38. Karthik R, Hafila MIF, Saravanan C, Priyadarsini P, Ashwath B. Assessing prevalence of temporomandibular disorders among university students: a questionnaire study. J Int Soc Prev Community Dent. 2017; 7: 24-29.

39. Huhtela OS, Näpänkangas R, Joensuu T, Raustia A, Kunttu K, Sipilä K. Self-Reported Bruxism and Symptoms of Temporomandibular Disorders in Finnish University Students. J Oral Facial Pain Headache. 2016; 30(4):311-317

40. Bair E, Gaynor S, Slade GD, et al. Identification of Clusters of Individuals Relevant to Temporomandibular Disorders and Other Chronic Pain Conditions: The OPPERA Study. Pain. 2016 Jun;157(6):1266-1278.

41. Moayedi M, Weissman-Fogel I, Crawley AP, et al. Contribution of chronic pain and neuroticism to abnormal forebrain gray matter in patients with temporomandibular disorder. Neuroimage. 2011 Mar $1 ; 55(1): 277-286$.

42. Damian RI, Spengler M, Sutu A, Roberts BW. Sixteen going on sixty-six: A longitudinal study of personality stability and change across 50 years. J Pers Soc Psychol. 2019;117(3):674-695.

43. Briley DA, Tucker-Drob EM. Genetic and environmental continuity in personality development: a metaanalysis. Psychol Bull. 2014;140(5):1303-1331.

44. Osma J, Suso-Ribera C, García-Palacios A, Crespo-Delgado E, Robert-Flor C, Sánchez-Guerrero A, Ferreres-Galan V, Pérez-Ayerra L, Malea-Fernández A, Torres-Alfosea, MA. Efficacy of the unified protocol for the treatment of emotional disorders in the Spanish public mental health system using a group format: study protocol for a multicenter, randomized, non-inferiority controlled trial. Health and quality of life outcomes. 2018; 16(1): 46. 
45. Sugawara N, Yasui-Furukori N, Sasaki G, et al. Coping behaviors in relation to depressive symptoms and suicidal ideation among middle-aged workers in Japan. J Affect Disord. 2012 Dec 15;142(13):264-268.

46. Carver CS, Scheier MF, Weintraub JK. Assessing coping strategies: a theoretically based approach. J Pers Soc Psychol. 1989 Feb; 56(2):267-283.

47. Slade GD, Oh rbach R, Greenspan JD, et al. Painful Temporomandibular Disorder: Decade of Discovery from OPPERA Studies. J Dent Res. 2016; 95(10):1084-1092.

48. Enns A, Eldridge GD, Montgomery C, et al. Perceived stress, coping strategies, and emotional intelligence: A cross-sectional study of university students in helping disciplines. Nurse Educ Today. 2018 Sep; 68: 226-231.

49. Por J, Barriball L, Fitzpatrick J, et al. Emotional intelligence: Its relationship to stress, coping, wellbeing and professional performance in nursing students. Nurse Educ Today. 2011 Nov; 31(8):855860.

\section{Figures}




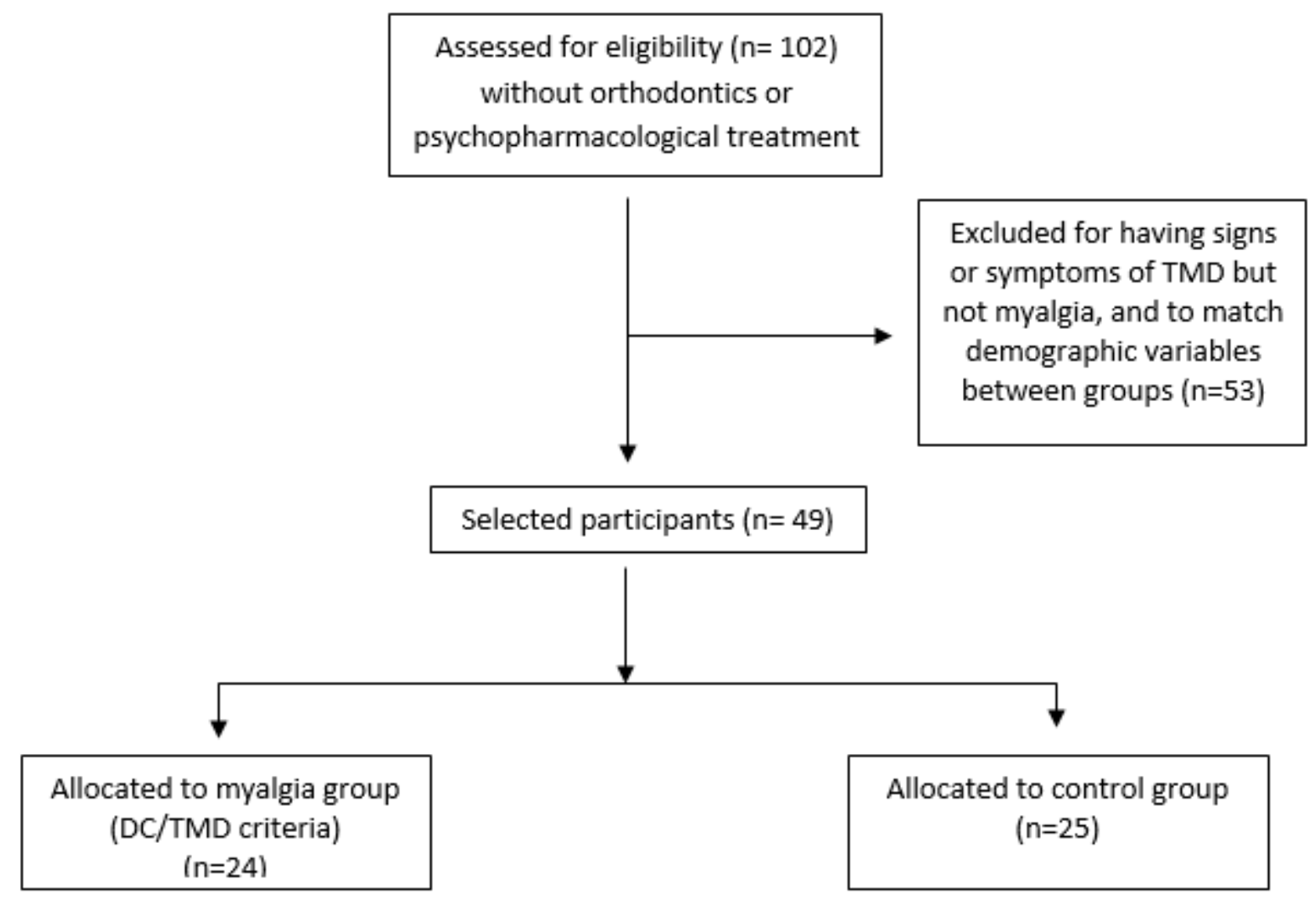

Figure 1

Consort-squeme of participant selection and eligibility criteria

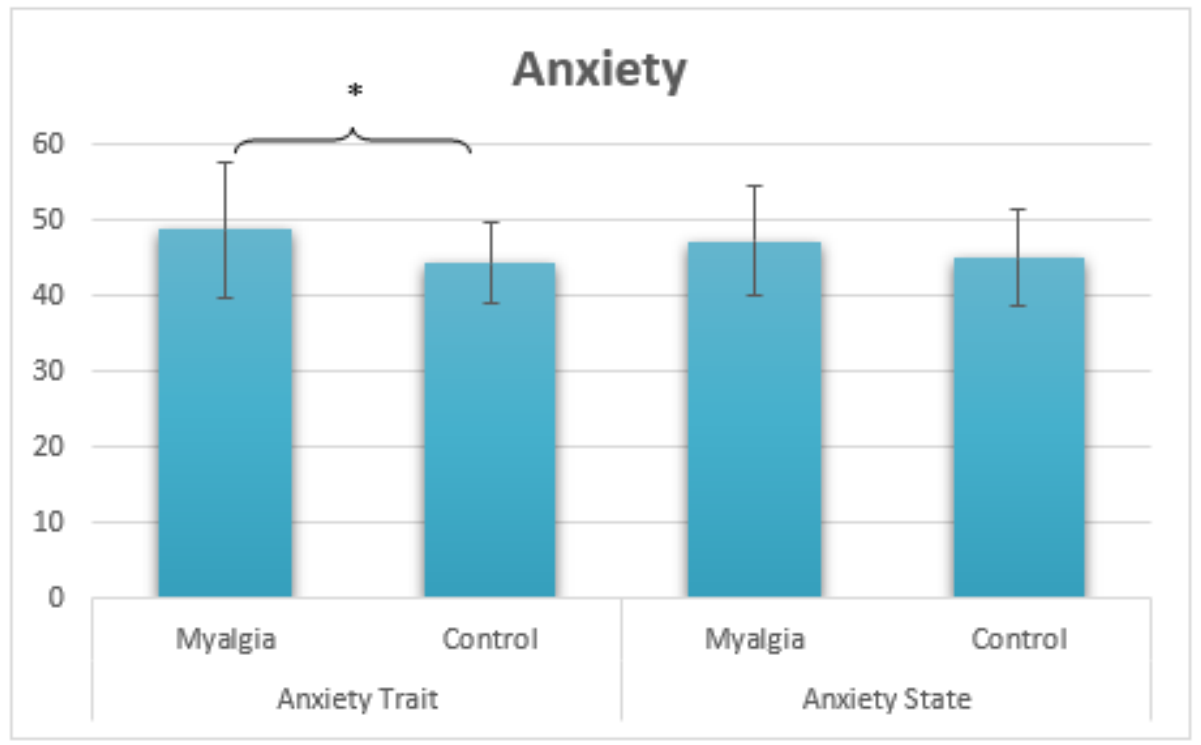

Figure 2 
State-Trait Anxiety Inventory results. (Graphic shows t-scores of the test). *: $p<.05$

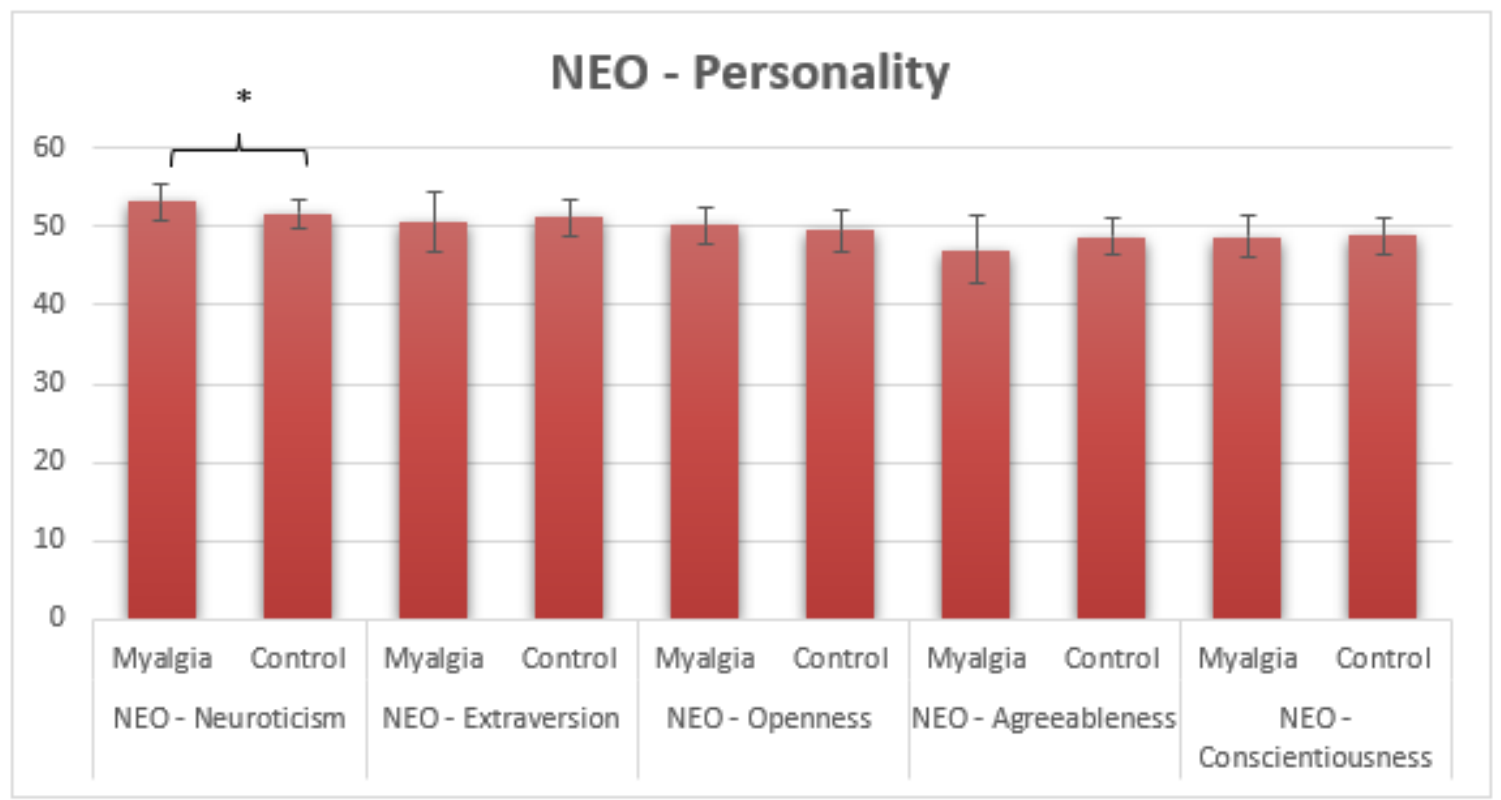

\section{Figure 3}

NEO five factor inventory results. (Graphic shows t-scores of the test). *: $p<.05$

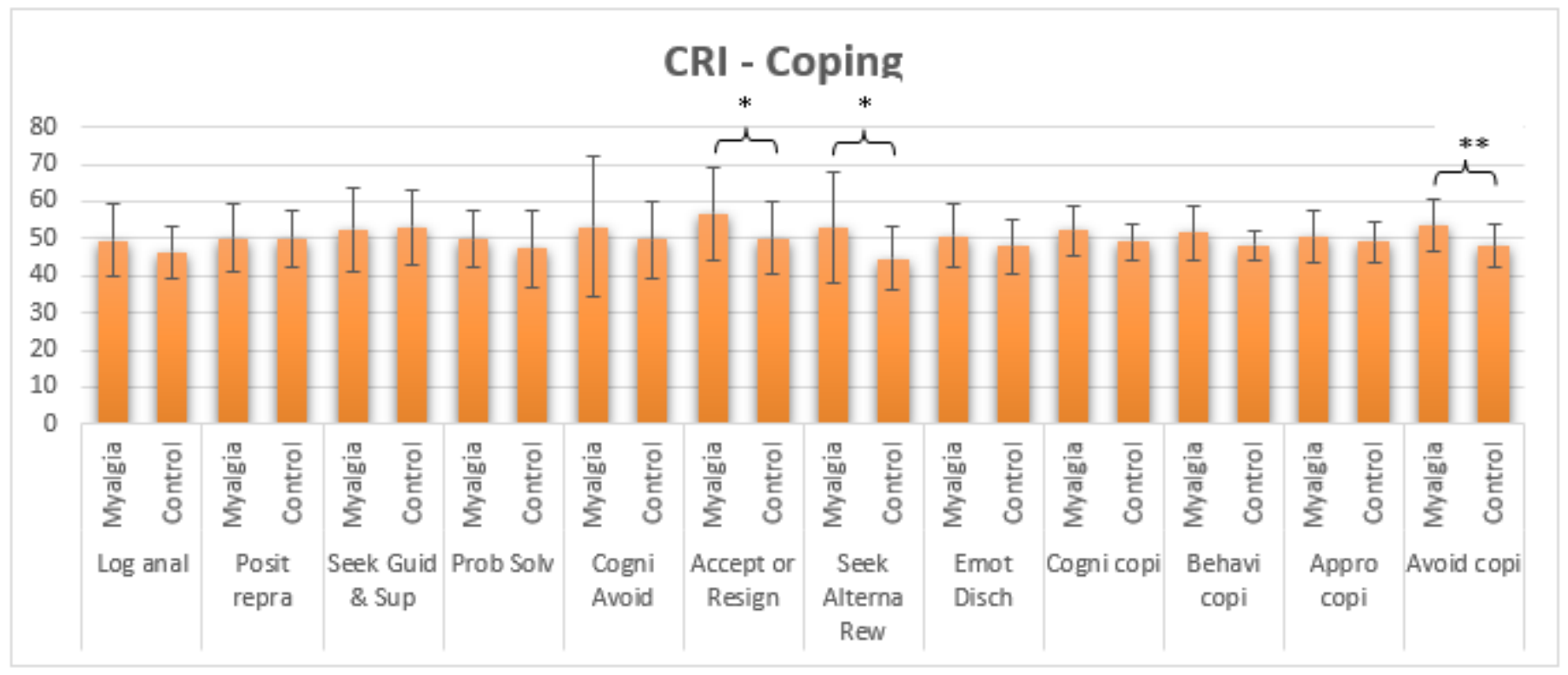

\section{Figure 4}

Coping responses inventory results. (Graphic shows t-scores of the test). *: $p<.05 ; * \star: p<.01$ 